\title{
DESAFIOS DA ATENÇÃO PRIMÁRIA À SAÚDE DE PESSOAS IDOSAS EM TEMPOS DE PANDEMIA
}

\section{Fabíola de Araújo Leite Medeiros'}

ORCID: 0000-0002-0834-1155

Ana Claudia Torres de Medeiros"

ORCID: 0000-0002-3695-9745

Rafaella Queiroga Souto'"' ORCID: 0000-0002-7368-8497

'Universidade Estadual da Paraíba. Campina Grande, Paraíba. Brasil. DCEG ABEn-PB.

"Universidade Federal de Campina Grande. Campina Grande, Paraíba. Brasil. DCEG ABEn-PB.

"'Universidade Federal da Paraíba. João Pessoa, Paraíba. Brasil. DCEG ABEn-PB

Autor Correspondente:

Fabíola de Araújo Leite Medeiros Email: profabiola@bol.com.br

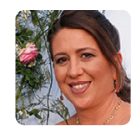

Como citar:

Medeiros FAL, Medeiros ACT, Souto RQ. Desafios da Atenção Primária à Saúde de Pessoas Idosas em Tempos de Pandemia. In: Santana RF (Org.). Enfermagem gerontológica no cuidado do idoso em tempos da COVID 19. Brasilia, DF: Editora ABen; 2021. 171 p. (Serie Enfermagem e Pandemias, 5). https://doi.org/10.51234/aben.21.e05.c02

\section{INTRODUÇÃO}

Desde o início do ano de 2020, a população mundial tem vivenciado uma situação atípica, a pandemia da Covid-19, que transformou a vida em decorrência do avançar de casos e da busca pelo seu controle. O mundo inteiro tem lançado mão de uma rotina, que, atualmente, foi denominada pelo termo'novo normal', pautado em cuidados sanitários para a prevenção da doença, enquanto não se chega ao controle eficaz por parte de vacinas ou medicamentos que se mostrem eficientes contra a versão da doença.

Considerando as iniciativas adotadas para o controle da pandemia, desde o reconhecimento dessa enfermidade no mundo, que autoridades públicas lançaram, principalmente para grupos vulneráveis, como as pessoas idosas, medidas ainda mais restritivas, como o distanciamento ou isolamento social rigoroso e aplicação de metas sanitárias duras em todos os setores sociais visando a diminuição de contágio no convívio social e execução de apenas atividades ditas essenciais à sobrevivência no período de pandemia. Sendo assim, escolas, universidades, academias, comércios e muitos dos serviços públicos foram fechados para o controle da pandemia.

O novo coronavírus se manifestou em um grande percentual da população, tendo gerado milhares de morte em todo o mundo. No Brasil, até o dia 09 de agosto de 2020, de acordo com dados do Ministério da Saúde, da população geral brasileira (210.028.613 indivíduos), houve registro de 101.049 óbitos por Covid-19, com registros oficiais de 3.035.422 casos notificados e acumulados ${ }^{(1)}$.

Tomando por base esse cenário, o Brasil se situou no ranking de mais de 100.000 mortes até agosto de 2020, havendo também registros oficiais de 1.209.208 pessoas recuperadas da doença. O número de pessoas curadas tem superado à quantidade de casos ativos (643.483). Apesar disto, esses dados não são suficientes para o controle sanitário da doença, considerando a gravidade da mesma, 
a ausência de tratamentos eficazes e o risco de mortalidade dentre os grupos vulneráveis. A doença está presente em $96,4 \%$ dos municípios brasileiros. Associado a pandemia do Covid-19, outros índices assustadores de arborviroses como a Dengue, a Chinkunguya e a Zica ainda assolam grande parte da população, doenças que apresentam sintomatologia que se confunde aos sintomas da covid-19(1).

Atrelada a doenças de ordem transmissíveis e de risco à saúde da população de pessoas idosas no Brasil, enfoca-se que o distanciamento ou isolamento social, necessário ao controle da pandemia da Covid-19, modificou a rotina de promoção ao envelhecimento ativo, que conduz a pessoa idosa à inserção social e à atividade física regular com hábitos de vida saudável, com fins para a boa funcionalidade corporal e mental.

A quebra na promoção do envelhecimento ativo e o descontrole da saúde da pessoa idosa, considerando os 'novos normais' embasados em distanciamento ou isolamento social, estão presentes dentre focos assistenciais nas agendas das entidades públicas em relação à saúde da população que envelhece, considerando que o público com idade igual ou superior aos 60 anos é muito acometido por Doenças Crônicas não Transmissíveis (DCNTs) que merecem atenção especial. Reconhece-se que distanciamento ou isolamento social tem sido uma das medidas essenciais e prioritárias em épocas de pandemia da Covid-19, mas precisa ser otimizada sem perder de vista, o que se tem feito até então, na programação das políticas públicas de saúde à pessoa idosa ${ }^{(2-3)}$.

O distanciamento ou isolamento social deveriam ter sido discutidos em âmbito político-ministerial, tendo em vista a própria Política Nacional da Saúde da Pessoa Idosa e dos documentos da Rede de Organização da Atenção em Saúde, em conformidade ao que reza as diretrizes do Modelo de Atenção à Saúde Integral da Pessoa Idosa, visando que o foco assistencial urgente fosse dado e aplicado socialmente, mas com menos riscos também de ageismo ou violência doméstica relacionada a própria medida preventiva em saúde ${ }^{(4)}$.

Ressalta-se que o reconhecimento da heterogeneidade e dos determinantes sociais na saúde do brasileiro são aspectos particulares na dinâmica de saúde que objetiva a promoção de um Modelo de Atenção Integral à Saúde da Pessoa Idosa no Brasil( ${ }^{(3)}$. Dessa forma, percebe-se que os perfis de idosos no Brasil e os arranjos de suporte de cuidado são diversos, principalmente quando determinados pela situação social e econômica dos brasileiros. Pessoas idosas que moram sozinhas, pessoas idosas que passaram a viver confinadas sem comunicação e/ou motivação pela vida, e sim amedrontadas e sem acesso aos meios tecnológicos que transmitem a informação necessária em tempos de pandemia.

Portanto, a questão norteadora que orientou a construção do presente capítulo foi: Quais os desafios da atenção primária à saúde (APS) para a manutenção da linha de Cuidado para as pessoas idosas, principalmente no âmbito do Sistema Único de Saúde e das redes de apoio social em tempos de pandemia da Covid-19? Não deixando de considerar que a pandemia é o foco prioritário dentre as ações mais urgentes, porém não se pode deixar de curso, principalmente na APS, a saúde integral da pessoa idosa com base na avaliação da funcionalidade e de condições clínicas pertinentes ao controle das DCNTs, o risco de exposição às arboviroses e às diversas outras patologias infectocontagiosas.

\section{OBJETIVO}

Tecer uma reflexão sobre os desafios da APS para a manutenção da linha de Cuidado com pessoas idosas em tempos de pandemia da Covid-19.

\section{MÉTODOS}

Tratou-se de um estudo teórico e reflexivo, usando do método de revisão narrativa, sobre os cuidados na APS e os desafios em tempos de pandemia da Covid-19.

Os dados analisados foram fundamentados nos documentos oficiais do Ministério da Saúde e de órgãos nacionais e internacionais em saúde, e de referencial bibliográfico sobre saúde e pandemia. As reflexões foram 
embasadas em artigos nacionais e internacionais, bem como nas vivências das autoras junto a APS e como membros especialistas nas ações programadas do Departamento Científico de Enfermagem Gerontológica da ABEn Sessão PB.

Assim, os resultados convergiram em duas categorias temáticas: 1) Atenção Primária à Saúde e a Pessoa Idosa; 2) Desafios da Atenção Primária à Saúde em tempos de Pandemia da Covid-19.

\section{ATENÇÃO PRIMÁRIA À SAÚDE E A PESSOA IDOSA}

Reconhece-se que o fenômeno do envelhecimento populacional no Brasil tem acontecido de forma rápida, em que até então, com expectativa de vida registrada até o ano de 2016, para ambos os sexos, numa média aproximada de 75,72 anos, sendo 79,31 anos para mulheres e 72,18 anos para homens. Considera-se que esse incremento na expectativa de vida do brasileiro é resposta às ações públicas principalmente, no âmbito da atenção básica, que tem determinado uma certa melhoria das condições de vida, com ampliação de visibilidade, e de acesso a serviços médicos preventivos e curativos, avanço da tecnologia médica, ampliação da cobertura de saneamento básico, aumento da escolaridade e da renda, entre outros determinantes sociais que indicam tal crescimento populacional, e principalmente, do incentivo a promoção do envelhecimento ativo e saudável ${ }^{(1-3,5-7)}$.

Ressalta-se que o Brasil é um país com desigualdade social e que há a necessidade de contínuo investimento de ações públicas para a melhoria das condições de saúde, para que tal conquista seja marcada por qualidade de vida de quem envelhece, assim como da diminuição de agravos à população em geral.

Apesar da pandemia da Covid-19, o Brasil não pode perder o foco da importância da atuação da APS na promoção a saúde da população, pois, os problemas preexistentes apenas foram evidenciados pela situação, demonstrando falhas no sistema público de saúde. Haja vista, que a Covid-19 se manifesta mais gravemente nos indivíduos acometidos pelas DCNTs, como Hipertensão, Obesidade, Diabetes e Câncer ${ }^{(8-10)}$.

A problemática reforçou a importância da APS no contexto no SUS, como fonte prioritária de do planejamento e execução de ações em saúde, principalmente relacionadas à promoção de saúde, prevenção de agravos e encaminhamentos de casos mais complexos para outros níveis de atenção à saúde, também do SUS(11).

Pesquisas de avaliação de serviços no Brasil que discorrem sobre a Estratégia Saúde da Família (ESF), como sendo uma intervenção positiva no campo dos serviços de saúde. Embora a ESF seja essencial na organização da Rede de Atenção em Saúde, na conjuntura atual tem enfrentado dificuldades de suporte técnico para um bom ajustamento junto às políticas públicas e incentivos governamentais que tem dificultado a boa atuação como porta de entrada do SUS. A busca da qualidade do cuidado tem sido uma tarefa complexa devido às crises políticas e financeiras que assolam o país nas recentes décadas associadas às dificuldades que englobam a falta de consensos sobre definições e planos de ações das políticas públicas, porém a luta dos profissionais e de pesquisadores nacionais que se preocupam com a saúde, com seus indicadores, desigualdades e determinantes sociais, tem sido fonte de resistência para dar continuidade às boas práticas e integralidade do cuidado em todos os níveis de atenção(12-13).

Associar a APS com o cuidado integral prestado às pessoas idosas, remete ao entendimento às políticas públicas de saúde, que inclui desde a Política Nacional de Promoção a Saúde, a Política Nacional de Humanização até a Política Nacional de Saúde da Pessoa Idosa. Faz-se referência também a todo o projeto de Diretrizes a Linha de Cuidado da Pessoa Idosa pelo SUS, na perspectiva do Modelo de Atenção à Saúde Integral(2-3,5-6). Os citados documentos oficiais determinam toda uma programação de saúde com vistas aos princípios básicos do SUS para a pessoa idosa, que são: a promoção do envelhecimento ativo e saudável; atenção integral; estímulo às ações intersetoriais; provimento de recursos para seguridade da assistência; formação e educação permanente dos profissionais de saúde do SUS na gerontologia; divulgação e informação sobre as políticas públicas voltadas às pessoas idosas e promoção de cooperações nacionais e internacionais das experiências na atenção à saúde $\mathrm{e}^{(2,5)}$. 
Em 2014, a Coordenação de Saúde da Pessoa Idosa do Ministério da Saúde publicou um documento que compunha as diretrizes para o cuidado das pessoas idosas no SUS, na perspectiva de lançamento de um Modelo de Atenção Integral, cujo objetivo foi orientar a organização do cuidado ofertado à pessoa idosa no âmbito do SUS como fortalecimento do plano integrado na Rede de Atenção à Saúde ${ }^{(3)}$.

Em 2018, foi lançado, pelo Departamento Programático e Estratégico da Secretaria de Saúde do Ministério, um documento sobre orientações técnicas para a implementação de linha de cuidado para atenção integral à saúde da pessoa idosa no sistema único de saúde - SUS, colaborando com estratégias de organização do setor saúde em ações benéficas a saúde integral, considerando as particularidades da pessoa idosa. Nesse documento, a APS entra como a porta de entrada do SUS e direciona toda a atenção de forma integral pautada na avaliação global da pessoa idosa, com olhar clínico-funcional dentro da rede de atenção em saúde ${ }^{(3)}$.

As Diretrizes do Modelo de Atenção Integral e as Orientações para a implementação da linha de cuidados da pessoa idosa incluem basicamente cuidados específicos a clientela, incluindo ações que acompanham desde o cadastro e o acolhimento humanizado da pessoa idosa e família a avaliação multidimensional e estratificação dos perfis das pessoas idosas quanto a funcionalidade e acometimento em saúde; até o encaminhamento e monitoramento de ações de cuidados críticos, de cuidados prolongados e de cuidados paliativos, seja em ambientes institucionais ou domiciliares ${ }^{(2-3)}$.

Verifica-se que os documentos de orientação a implementação da linha de cuidados à pessoa idosa no SUS, inicia-se na atenção básica, considerando que os documentos se orientam no cuidado integral ao indivíduo, família e comunidade, utilizando como porta de entrada do SUS, a APS. Unificando que as iniciativas essenciais voltadas ao cuidado ao grupo de pessoas idosas no âmbito do SUS se fundamentam na avaliação clínico-funcional, com base maior, na manutenção da funcionalidade como prerrogativa essencial dos programas de promoção ao envelhecimento ativo e saudável. A estratificação de perfis de idosos independentes, dependentes parciais e dependentes de terceiros para execução de atividades de vida diária é meta essencial dentro da perspectiva do plano de ação de cuidados. Há o que se avaliar em termos de sinais clínicos de alerta que poderão comprometer a saúde do idoso, que estão relacionados diretamente às condições clínicas (crônicas e agudas) ${ }^{(3)}$.

Reconhece-se que o perfil epidemiológico da população idosa é caracterizado pela tripla carga de doenças com forte predomínio das condições crônicas, prevalência de elevada mortalidade e morbidade por condições agudas decorrentes de causas externas e agudizações de condições crônicas e possivelmente infecções agudas ${ }^{(14)}$.

Um dos recursos utilizados dentre as orientações para a APS em relação à pessoa idosa, diz respeito ao exercício da clínica ampliada com utilização da ferramenta da Caderneta do Idoso e do Projeto Terapêutico Singular (PTS), visando uma estratégia de cuidado que articula um conjunto de ações integradas na construção coletiva e da singularidade de cada situação sobre a qual o projeto terapêutico atua. De acordo com os documentos oficiais que norteiam as atenções de saúde às pessoas idosas, estes sempre trazem dentre as citações a especificidades desse grupo etário, as quais extrapolam compreensões e condutas utilizadas no contexto da população adulta geral ${ }^{(2-3)}$.

É essencial que o profissional de saúde em todos os níveis da atenção, mas principalmente na APS, reconheça que a senescência traz consigo perda progressiva e lenta da reserva homeostática, além da heterogeneidade marcada pelas diferentes formas de envelhecer, determinando uma maneira peculiar de cuidar das afecções a saúde com base nos diferentes aspectos de apresentação, instalação e desfecho de doenças e agravos em saúde, levando a maior risco de vida e vulnerabilidade a eventos adversos, necessitando de ações multidimensionais e multissetoriais com foco no cuidado(2).

Nesse contexto, percebe-se o quão relevante é a atuação da APS no cuidado de pessoas idosas, e que, embora as iniciativas de orientação para a linha do cuidado integral à pessoa idosa sejam consideradas recentes, pois se configuram como proposta a partir do ano de 2018, essa deve ser perseverada como molde 
para reorganização da atenção em saúde e do desenvolvimento de planos terapêuticos mais eficazes às demandas populacionais em tempos de envelhecimento populacional, diminuindo os riscos de incapacidades e mortes precoces em pessoas idosas.

\section{DESAFIOS DA ATENÇÃO PRIMÁRIA À SAÚDE EM TEMPOS DE PANDEMIA DA COVID-19}

A pandemia da Covid-19, foi responsável pela adoção de medidas sanitárias de segurança e prevenção do contágio do coronavírus, e o foco assistencial prioritário se voltou aos casos confirmados, havendo uma paralisação da execução dos diversos setores sociais, e sem sombras de dúvidas, no setor da saúde houve abaulamento nas condutas que orientam, até então, o plano estratégico de implementação de ações públicas relacionados ao Modelo de Atenção Integral à Pessoa Idosa.

O confinamento domiciliar, principalmente das pessoas idosas, dificultou o acesso da APS na continuidade de ações programáticas para implementação da linha de cuidado integral pessoa idosa. E esse é um dos grandes desafios da ESF e da APS para contornar os danos ocasionados em época de pandemia.

É notório que o'novo normal', que é a convivência com normas sanitárias mais duras e precisas em tempos de pandemia da Covid-19 e, consequentemente o distanciamento e isolamento social, são essenciais para a provisão do controle sanitário da doença até que se tenha segurança com tratamentos eficazes e vacinas preventivas ${ }^{(10)}$. Porém, é essencial, que após reajustes dessas conformidades de manutenção de distanciamento social não haja distanciamento de cuidados.

Logo, os desafios para as equipes de APS refletem em ajustes técnicos de como traçar metas em prol do acolhimento, cadastramento de pessoas idosas, da estratificação desta população por meio dos diferentes perfis de funcionalidade, monitorar a rede de apoio à pessoa idosa, seja ela o apoio familiar, solidário e/ ou institucional e determinar metas essenciais para a supervisão e otimização do envelhecimento ativo e saudável(4,10,15-16).

Dentre a organização das Redes de Atenção à Saúde, a APS exerce uma função primordial com foco especial nas ações da ESF, na ampliação do acesso, melhora dos indicadores de saúde e na redução das desigualdades sociais e regionais. A pandemia impulsionou um solapamento de ações de promoção à saúde pela APS, e foi reforçada pela crise política e econômica, evidenciada pelas medidas adotadas como prioritárias pelo governo neoliberal, que coloca o investimento no setor saúde como um plano secundário ${ }^{(16)}$. Portanto, nota-se que assemelha-se tudo em torno da pandemia da Covid-19 e, que os demais agravos de saúde são desnecessários no presente momento por qual a população vivencia, o que denotaria uma falta de planejamento. Um outro desafio para APS vai surgindo, traçar medidas, que vão além do controle da pandemia, restabelecer as condutas contínuas de promoção de saúde junto aos determinantes sociais e principalmente, com o distanciamento social.

O fechamento temporário de parques, academias, comércio e dos demais setores sociais num período prolongado de meses afetou significativamente às ações intersetoriais de saúde voltadas a promoção de um envelhecimento ativo. Sendo assim, muitos documentos e protocolos estão sendo viabilizados por organizações nacionais e internacionais de saúde, assim como, órgãos públicos e privados, como fontes de comunicação para seguimento de regras viáveis para organização dos setores de saúde.

Por exemplo, a ABRASCO em 2020 elaborou um documento e veiculou em rede nacional como meio a orientar a APS e ESF no enfrentamento de ações junto a pandemia, e nesse material, há menção de condutas com grupos vulneráveis como o das pessoas idosas. O documento respalda-se que dependendo de cada contexto, de cada Unidade Básica de Saúde e de cada município, deverá haver o diálogo e articulação entre gestores e trabalhadores de saúde através do Conselho Nacional de Saúde e demais conselhos para tornar efetivo o fortalecimento da APS no SUS(16).

Com base nesse documento e em outros publicados até agosto de 2020, é possível elencar ações desafiadoras na APS em relação ao cuidado integral à pessoa idosa, tais como: desenvolver ações de vigilância em 
saúde para bloquear e reduzir o risco de expansão da Covid-19, com apoio ao isolamento social e quarentena de casos e contatos; promoção de educação em saúde, com uso da linha de cuidado por telefone específico para atenção aos sintomáticos e acompanhamento diário; separar o fluxo de atenção dos sintomáticos respiratórios/casos suspeitos de Covid-19 do fluxo de pacientes com outros problemas/necessidades; oferecer suporte a grupos mais frágeis e vulneráveis que necessitarão de atenção especial; a equipe do território cabe coordenar e monitorar as ações dirigidas à população sob sua responsabilidade, em articulação com os serviços especializados e complementares; incentivar à APS a enfrentar o isolamento e a quarentena com idosos independentes, parcialmente dependentes e dependentes; além de demais pessoas acamadas e com múltiplas incapacidades; monitorar pessoas idosas portadoras de doenças crônicas e incapacidades em relação manutenção de hábitos de vida saudáveis mesmo em ambientes domiciliares; contactar com os cuidadores de idosos na manutenção de boa comunicabilidade e apoio aos cuidados junto a pessoa idosa e otimizar ações de educação em saúde com vistas ao envelhecimento ativo e saudável em meios tecnológicos pactuados junto às ações de cuidado para que mesmo sob distanciamento físico, a pessoa idosa não se sinta distanciada da unidade básica de saúde ${ }^{(1,10,15,16)}$.

Apesar dos desafios, fica evidenciada a necessidade de planejamento para o retorno paulatino das atividades, inclusive na perspectiva presencial. A APS precisa manter ativas suas ações essenciais na linha de cuidado à pessoa idosa, sem se tornar, um agente promotor da disseminação do vírus neste grupo etário.

\section{CONSIDERAÇÕES FINAIS}

Os desafios da APS diante do cuidado integral à saúde de pessoas idosas em tempos da pandemia da Covid-19, são consequência das medidas contingenciais adotadas pelos governos, como o distanciamento ou isolamento social, em decorrência da ausência de vacinas e tratamentos específicos ao combate da doença e seu contágio.

Entre eles, destaca-se a dificuldade da implantação da linha de cuidados integrais junto a essa população, que requer estratégias de aproximação e envolvimento com o indivíduo idoso, sua família e comunidade. Outros desafios são pautados na crise política e econômica do sistema neoliberal, que dificulta a execução de políticas públicas junto a pessoa idosa, no que tange ações pautadas dos determinantes e condicionantes sociais do processo saúde-doença.

Tais desafios precisam estar claramente descritos nos documentos oficiais, nacionais e internacionais, com sugestões de iniciativas peculiares para o enfrentamento de tais dificuldades em tempos de pandemia.

Sugere-se que a APS, como porta de entrada do setor saúde, se reorganize em relação às iniciativas de atenção à saúde diante do'novo normal', não deixando de lado sua ação essencial de otimização dos índices de expectativa de vida, na segurança do cuidado integral junto à pessoa idosa e principalmente na prevenção de mortes precoces em pessoas que envelhecem no Brasil e no mundo.

\section{AGRADECIMENTO}

Ao Departamento Científico de Enfermagem Gerontológica da ABEn Nacional.

\section{REFERÊNCIAS}

1. Ministério da Saúde (BR). Informa SUS. Covid-19: Brasil já tem mais de 1,2 milhões de curados [Internet]. Brasília: DF. [publicado em 14 de Julho de 2020] [cited 2020 Jul 31] Available from: http://www.saude.gov.br/noticias/ agencia-saude/47202-covid-19-brasil-ja-tem-mais-de-1-2-milhao-de-curados.

2. Ministério da Saúde (BR). Diretrizes para o cuidado das pessoas idosas no SUS: proposta de Modelo de Atenção Integral[Internet]. XXX Congresso Nacional de Secretarias Municipais de Saúde. Brasília: DF, 2014[cited 2020 Jul 31]. Available from: https://www.saude.gov.br/images/pdf/2019/abril/05/diretrizes-cuidado-pessoa-idosa-sus.pdf 
3. Ministério da Saúde (BR). Secretaria de Atenção à Saúde Departamento de Ações Programáticas e Estratégicas. Orientações técnicas para a implementação de Linha de Cuidado para Atenção Integral à Saúde da Pessoa Idosa no Sistema Único de Saúde - SUS [Internet]. 2018[cited 2020 Jul 31]. Available from: http://bvsms.saude.gov.br/bvs/ publicacoes/linha_cuidado_atencao_pessoa_idosa.pdf

4. Hammerschmidt KSA, Santana RFa. Health of the older adults in times of the Covid-19 pandemic. Cogitare Enferm. 2020;25:e72846. https://doi.org/10.5380/cev25i0.72849

5. Ministério da Saúde (BR). Política Nacional de Saúde da Pessoa Idosa [Internet]. Brasília: DF, 2006[cited 2020 Jul 31]. Available from: https://bvsms.saude.gov.br/bvs/saudelegis/gm/2006/prt2528_19_10_2006.html

6. Ministério da Saúde (BR). Estatuto do Idoso[Internet]. Brasília: DF, 2003[cited 2020 Jul 31]. Available from: http://www. planalto.gov.br/ccivil_03/leis/2003/l10.741.htm

7. Instituto Brasileiro de Geografia e Estatística (IBGE). Projeções em 2019[Internet]. 2020[cited 2020 Jul 31]. Available from: https://www.ibge.gov.br/estatisticas/sociais/populacao.html

8. Ministério da Saúde (BR). Boletim 4. Infecção Humana pelo Novo Coronavírus (2019-nCoV). [Internet]. 2020[cited 2020 Jul 31]. Available from: https://www.saude.gov.br/images/pdf/2020/fevereiro/04/Boletim-epidemiologico-SVS-04fev20.pdf 4

9. Ministério da Saúde (BR). Portaria MS/GM n. 188, de 3 de fevereiro de 2020. Declara Emergência em Saúde Pública de importância Nacional (ESPIN) em decorrência da Infecção Humana pelo novo Coronavírus (2019-nCoV) [Internet]. Diário Oficial da União, Brasília (DF), 4 de fevereiro de 2020 [cited 2020 Jul 31]. Seção 1:1. Available from: http://www.in.gov.br/ web/dou/-/ portaria-n-188-de-3-de-fevereiro-de-2020-241408388

10. World Health Organization (WHO). Statement on the second meeting of the International Health Regulations (2005) Emergency Committee regarding the outbreak of novel coronavirus (2019-nCoV) [Internet]. 2020 [cited 2020 Mar 27]. Available from: https://www.who.int/news-room/detail/30-01-2020-statement-on-the-secondmeetingof-theinternational-health-regulations-(2005)-emergency-committee-regarding-the-outbreak-of-novelcoronavirus-(2019nov)

11. Facchini LA, Dilélio AS. Qualidade da Atenção Primária à Saúde no Brasil: avanços, desafios e perspectivas. Saúde Debate. 2018;42(1):208-223. https://doi.org/10.1590/0103-11042018S114

12. Paim JS. Sistema Único de Saúde (SUS) aos 30 anos. Ciênc Saúde Coletiva [Internet]. 2018;23(6):1723-8. https://doi. org/10.1590/1413-81232018236.09172018

13. Castro M, Massuda A, Almeida G, Menezes-Filho NAM, Andrade MV, Noronha QVMS, et al. Brazil's unified health system: the first 30 years and prospects for the future. Health Policy. Lancet. 2019;394(10195). https://doi.org/10.1016/ S0140-6736(19)31243-7

14. Marinho F, Passos VMA, França EB. Novo século, novos desafios: mudança no perfil da carga de doença no Brasil de 1990 a 2010. Epidemiol Serv Saúde [Internet]. 2016[cited 2020 Aug 11];25(4):713-24. https://doi.org/10.5123/ S1679-49742016000400005

15. Sociedade Brasileira de Geriatria e Gerontologia (SBGG). Posicionamento sobre Covid-19 [Internet]. 2020[cited 2020 Jul 31]. Available from: https://sbgg.org.br/ posicionamento-sobre-covid-19-sociedade-brasileira-de-geriatria-e-gerontologia-sbgg-atualizacao-15-03-2020/

16. Associação Brasileira de Saúde Coletiva (ABRASCO). Fortalecer a Estratégia Saúde da Família no enfrentamento da Covid-19: posicionamento da Rede APS, da ABRASCO. 5 de maio de 2020. 2020 [cited $2020 \mathrm{Jul}$ 31]. Available from: https://www.abrasco.org.br/site/noticias/especial-coronavirus/ fortalecer-a-estrategia-saude-da-familia-no-enfrentamento-da-covid-19/47785/ 